\title{
Undiagnosed pheochromocytoma; a dilemma to the surgeon and the anaesthetist
}

\author{
Nishan tha JLAR, Perera SCR, Thilini HGD, Wijesinghe H, Iresha PV, Lankage W, Amarasooriya AMDB
}

Oncosurgery Unit, Teaching Hospital Karapitiya, Sri Lanka.

\author{
Correspondence: Dr. Ruwan Nishantha \\ e-mail: ruwanjaya2@gmail.com \\ D https://orcid.org/0000-0002-1330-7971 \\ Dr. Iresha P Vithanage \\ e-mail: ireshapv@gmail.com \\ (D) https://orcid.org/0000-0001-7099-3210 \\ Submitted on 17.06.2021 and accepted for publication on 04.08.2021
}

\section{Introduction}

Pheochromocytoma (PCC) is a catecholaminesecreting tumor in the adrenal medulla and paragangliomas (PPGL) are tumours arising from the chromaffin cells of the sympathetic and parasympathetic chains. PCCs are rare neuroendocrine tumours with incidence of 1-2 : 100,000

Usually adrenal medullary tumours and PPGL arising from the sympathetic chain are secretory; while tumours of parasympathetic counterpart are confined to the head and neck are nonsecretory. Secretory PCC and PPGL produce the catecholamines epinephrine, norepinephrine and less frequently dopamine or their metabolites metanephrine, normetanephrine and methoxytyramine.

It is also called as the "tumour of $10 \%$ ", being $10 \%$ extra-adrenal, $10 \%$ familial, $10 \%$ in children, $10 \%$ being bilateral and $10 \%$ being malignant. In the hereditary form, PCC is a part of more complex clinical syndrome whose causative genes have been identified. The most common causes of PCC and PPGL consist with multiple endocrine neoplasia type 2A and 2B (MEN2A/ 2B), Von Hippel Lindau disease (VHL), neurofibromatosis type 1 (NF1) and familial pheochromocytoma/ paraganglioma syndromes.

Secretory PCC typically presents as a sustained or paroxysmal hypertension, headache, sweating, palpitations, weight loss and pallor. These symptoms can be aggravated in certain situations such as induction of anaesthesia, tumour manipulation, micturition and with particular medications like tricyclic antidepressants, metoclopramide, tyramine and glucagon.

The diagnosis of pheochromocytoma usually requires biochemical evidence of excessive catecholamine production by the tumour. Usually it is obtained by measuring catecholamines or catecholamine metabolites in the urine and plasma. Plasma free metanephrine level is considered as a gold standard than 24 hour urinary fractionated metanephrine level or urinary vanillylmandelic acid (VMA) level. These biochemical tests should be recommended, if a PCC is clinically suspected or incidentally found to have an adrenal mass. Mortality is high in patients with secretory PCC or PPGL undergoing surgery without pre-operative medical therapy.

Alpha adrenergic blockade, a beta blocker and liberal intake of salt are recommended for all secreting PCC or PPGL. Medication starts 10 - 14 days before surgery to reduce the mortality associated with removal of these tumours. However, if biochemical testing is negative, it is a common practice to not to perform pre-operative medical preparation. 
We report a case with biochemically negative right adrenal tumour, but it complicated her first pregnancy and behaved as a secretory pheochromocytoma at the induction of general anaesthesia and during mobilisation of the tumour.

\section{Case report}

A 22-year-old primigravida was diagnosed to have pregnancy induced hypertension (PIH) during the third trimester of her pregnancy. She was treated with oral labetalol and followed up with frequent blood pressure monitoring.

At 32 weeks of gestation, patient was admitted to hospital due to elevated blood pressure and an emergency caesarean section was performed due to resistant hypertension. Intraoperative and postnatal period was uneventful except persistently elevated blood pressure, despite giving regular oral hypertensives: calcium channel blockers (nifedipine) and angiotensin-conversion enzyme inhibitors (captopril). Then the patient was evaluated for young hypertension and ultrasound scan abdomen revealed a right adrenal mass. In CECT abdomen and pelvis, she was found to be having a $6.1 \times 4.8 \times 4.3 \mathrm{~cm}$ well defined right adrenal mass with enhanced attenuation. The left adrenal appeared to be normal.

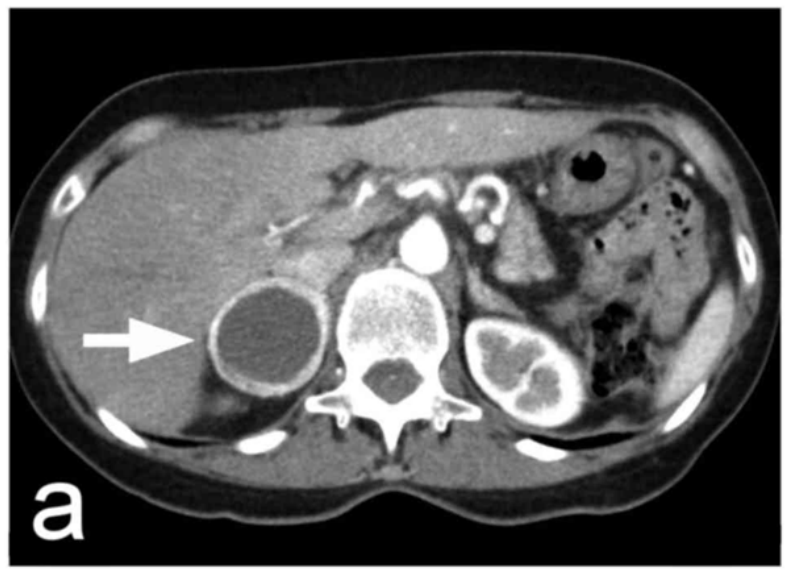

Figure 1: The appearances of the MRI abdomen and pelvis favoured a malignant right adrenal neoplasm

Retrospectively, she denied any symptoms except infrequent episodes of palpitations. On physical examination, blood pressure was $130 / 100 \mathrm{mmHg}$ without postural hypotension and pulse rate was 110 bpm. Her physical examination was unremarkable except resting tachycardia.

Her 24-hour urinary metanephrine excretion was assayed twice and the values were within the reference range on both occasions; $62.95 \mu \mathrm{g} / 24 \mathrm{hrs}$ and $72.8 \mu \mathrm{g} / 24 \mathrm{hrs}$ respectively (normal $\leq 330 \mu \mathrm{g} /$ $24 \mathrm{hrs}$ ). Overnight dexamethasone suppression test was appropriate with suppressed AM cortisol of $32.9 \mathrm{nmol} / \mathrm{L}$. Serum aldosterone level was $7 \mathrm{ng} / \mathrm{dL}$ and plasma renin activity was $12.2 \mathrm{ng} / \mathrm{mL} /$ hour (ratio, $\leq 20$ ). In addition, 24 hour ambulatory blood pressure monitoring was performed and it illustrated increased day and night average blood pressure.

With the normal biochemical measurements, she was diagnosed as having a non-secretory PCC.

However, a selective alpha-1 adrenoceptor blocker (prazosin $0.5 \mathrm{mg}$ tds) was started to reduce the blood pressure. Right side adrenalectomy was scheduled once the blood pressure became normal.

Surgery was performed two weeks after starting prazosin and during induction of general anaesthesia patient developed tachycardia (pulse rate $-140 \mathrm{bpm}$ ) and she was treated with intravenous drugs and pulse rate dropped to100 bpm subsequently. In exploratory laparotomy a well-defined right adrenal tumour was found. While handling the tumour, once again the vital cardiovascular parameters altered significantly. Pulse rate rose up to $160 \mathrm{bpm}$ and pressure shot up to $220 / 160$ mmHg. With the administration of intravenous antihypertensives, she became profoundly hypotensive, requiring vasopressors and intravenous fluids. She was resuscitated and patient became stable soon after the removal of tumour.

Post-operative care was given at the intensive care unit and the postoperative period was uneventful. She was normotensive throughout the post-operative period and discharged on postoperative day 5 without any antihypertensive treatment. She was followed up at clinic regularly and histopathology revealed the mass to be a benign pheochromocytoma. 


\section{Discussion}

The case under discussion is on a 22-year-old female in her first pregnancy who initially presented with PIH in the last trimester and underwent urgent section due to resistant hypertension. She was investigated further during the postpartum period due to persistently elevated blood pressure. Subsequently, it revealed a biochemically negative right adrenal tumour which behaved like a secretory tumour intraoperatively.

Pheochromocytoma can mimic the symptoms and signs of PIH and therefore diagnosis is often missed. However, PIH is usually accompanied by proteinuria, sudden weight gain and oedema as well as liver and coagulation abnormalities.

In hypertension associated with PCC, excessive urinary protein excretion and liver or coagulation abnormalities occur rarely and sudden weight gain and oedema are unusual (1).

In cases reported between 1980-87, maternal and foetal mortality rates were $33 \%$ and $39 \%$ respectively, when diagnosis was not made before the labour (2).

Screening should be undertaken in those with accelerated or progressive hypertension; hypertension occurring at a young age or not responding to conventional treatment and those with pressor responses to induction of anaesthesia, intravenous contrast media or labour (3).

Before alpha blockers were introduced in 1950, perioperative mortality, currently limited to $0-6 \%$ ranged between $24-50 \%$. Selective alpha 1 blockers were used to avoid side effects of non-selective alpha 1 blockers. Alpha-1 blockers do not prevent hypertensive crisis, but achieve better blood pressure and heart rate control (4).

Definitive treatment of PCC is surgical resection of the mass which is considered to be curative in $90 \%$ of the cases.

In a case of sustained paroxysmal hypertension or paradoxical hypertension despite antihypertensive therapy, especially during therapy with beta blockers, the diagnosis of pheochromocytoma has to be considered. (4)

This case report is based on a biochemically negative adrenal mass that clinically behaved like a secretory PCC with the induction of anaesthesia. While clinical and radiological signs were suggestive of possible PCC, her 24 hour urine metanephrine excretion was normal in two occasions. However, additional biochemical testing with plasma metanephrines or serum methoxytyramine would have helped us to absolutely rule out PCC.

Any patient with symptoms suggestive of PCC and all adrenal nodules should be screened for PCC. Urinary and plasma catacholamines, urinary and plasma metanephrines and urinary VMA test have been used to diagnose PCC and PPGL in the past. However, the sensitivity of plasma free metanephrines is highest at $99 \%(95 \% \mathrm{CI}$, $96 \%-100 \%)$ with a specificity of $89 \%(95 \% \mathrm{CI}$, $87-92 \%)(5,6)$ followed by urinary fractionated metanephrines with a sensitivity of $97 \%(95 \%$ CI, $92 \%-99 \%)$ and specificity of $69 \% .(95 \%$ CI, 64\% - 72\%) (5). Currently recommended initial screening test is measurement of plasma free metanephrines or 24 hour fractionated urinary metanephrines and once bio-chemical diagnosis is confirmed, localization is indicated with the aid of CT or magnetic resonance imaging (MRI). $\mathrm{CT}$ is the preferred initial imaging tool as opposed to MRI imaging with a reported sensitivity between 88 and 100\%. Benign adenomas are homogenous with unenhanced CT attenuation values of $<10$ Hounsfield Units (HU) along with rapid contrast medium washout of $>50 \%$ after 10 mins. Whereas, PCCs and malignant tumours usually have unenhanced attenuation of $>20 / \mathrm{hu}$ with delayed washout time $<50 \%$ (7).

Even asymptomatic patients with plasma or urinary metanephrines more than two to three times 7 normal should be evaluated for screening of PCC or PPGL.

In the past, most of the adrenal tumours were discovered during unrelated surgical operations or at autopsy. With the advancement in imaging, number of incidentally noted adrenal masses has risen.

Nonfunctioning PCCs are described by Simpson et al., (8) and Cevitt et al., (9). Both patients had presented with right sided abdominal pain and were found to have retroperitoneal masses with high vascularity. Masses were removed without 
biochemical evaluation prior to surgery and pathology showed benign PCC and epinephrineproducing PCCs respectively. Kota et al., (10) described biochemically silent PCC which clinically behaved like a secretory tumour despite a negative biochemical work up. An asymptomatic woman who was incidentally noted to have an adrenal mass with normal 24-hour urinary VMA and plasma metanephrines developed a hypertensive crisis during the surgical resection. The mass was removed 2 weeks later following treatment with alpha blockers. Mannelli et al., described a nonsecreting PCC in a woman found to have a right sided mass in an abdominal CT along with positive meta iodo benzyl guanidine (MIBG) scan, but normal urinary VMA. She was asymptomatic and uncomplicated surgical removal of this mass was done and histology came as PCC.

But there are case reports with non-classical presentations of PCC despite comprehensive laboratory testing and imaging techniques. These presentations are named as clinically unsuspected (11), asymptomatic (12), biochemically silent (10) non-functioning $(8,9)$ and non-secreting.

There are case reports related to non-secreting tumours with variably performed biochemical testing and inconsistency in definition. The inconsistency encountered in the different presentations is mainly due to variability of catecholamine secretion and metabolism of individual tumours (13). PCC and PPGL can have different secretory patterns (epinephrine, norepinephrine, dopamine or other metabolites) and appropriate hormones should be tested for the diagnosis. PCCs that are adrenergic or secrete metanephrines can be silent and present with paroxysmal crisis due to the down-regulation of adrenoceptors secondary to continuous high concentration of norepinephrine. At the same time, noradrenaline or normetanephrine secreting tumours can have co-existing hypertension (13).

Chen (14) described a 75-year-old asymptomatic man who had a past history of hypertension, with a right adrenal mass and normal urinary fractionated catecholamines and VMA. He had uncomplicated surgery without alpha blockade and pathology showed PCC.
In most of these cases, tumours were described as silent without evaluating preoperatively with biochemical testing. In other instances the biochemical testing was less sensitive/ specific than the currently recommended plasma free or 24 hour urinary fractionated metanephrines (15). In this case, the tumour behaved as a secreting tumour despite a negative biochemical workup. At the time of induction of anaesthesia alpha blockade has been shown to reduce mortality associated with surgery in patients with PCCs. Hence it may be important to perform additional biochemical testing in patients with a single negative test and a high suspicion for PCC on clinical grounds. In this case, further evaluation with serum methoxytyramine and 24 hour urinary metanephrine excretion would have been helpful.

It is important to determine whether preoperative alpha blockade is indicated or not, prior to surgical removal of adrenal mass considering combination of clinical, biochemical and radiological factors.

\section{Conclusion}

We present a case of a patient with an adrenal mass with high $\mathrm{HU}$ on $\mathrm{CT}$ and negative 24 hour urinary metanephrines, that behaved clinically like a secreting PCC during induction of anaesthesia and handling of tumour despite of prior treatment with alpha blockade.

Therefore, we should consider additional biochemical evaluation including testing both plasma and urinary metanephrines and serum methoxytyramine in cases with high clinical suspicion for PCC with negative initial biochemical tests.

It is better to consider alpha blockade in patients whom, preoperative clinical suspicion of secretory $\mathrm{PCC} / \mathrm{PPGL}$ is high irrespective of negative initial biochemical work up.

\section{References}

1. Oliva R, AngelosP, KaplanE, BakrisG, Pheochromocytoma in pregnancy: a case series and review. Hypertension. 2010; 55: 600-6. Epub January 18,2010. 
2. Harper MA, Murnghan CA, Kennedy L, Hadden DR, Atkinosoy AB. Pheochromocytoma in pregnancy. Five cases and a review of literature. Br J Obstet Gynaecol. 1989; 96: 594-606.

3. Platts JK, Drew PJ, Harrcy JN, Death from pheochromocytoma: lessons from a post-mortem survey. J Roy Coll Physicians Lond. 1995; 29: 299-306.

4. Reisch N, Peczkowskam, Januszewicz A, Neumann HP. Pheochromocytoma: Presentation, diagnosis and treatement. JHypertens. 2006; 24: 2331-9.

5. Lenders JW, Pacak K, Wulther MM, et al. Biochemical diagnosis of Pheochromocytoma: which test is best? JAMA. 2002; 287: 1427-1434.

6. Pacak K, Linenhan WM, Eisenhofer G, Walther MM, Goldstein DS. Recent advances in genetics, diagnosis, localization, and treatment of pheochromocytoma. Ann Intern Med. 2001; 134: 135-329.

7. Nieman LK. Approach to the patient with an adrenal incidentaloma. J Clin Endocrinol Metab. 2010; 95(9): 4106-4113.

8. Simpson ET, Murszalet WW, Ramsaroop R, Nonfunctioning pheochromocytoma. Urology.1985; 25: 632-633.
9. Levitt RG, Stanley RJ, Dehner LP. Angiography of a clinically non-functioning pheochromocytoma. Case report and review of the Literature. JAMA. 1975; 233: 268-269

10. Kota SK, Kota SK, Panda S, Modi KD. Pheochromocytoma on uncommon presentation of an asymptomatic and biochemically silent adrenal incidentaloma. Malays JMed Sci. 2012; 19(2): 86-91.

11. Sutton M, Sheps SG, Lie JT. Prevalence of clinically unsuspected pheochromocytoma. Review of a 50 year autopsy series. Mayo Clinic Proc. 1981; 56: 354-360.

12. Taubman I, Pearson OH, Anton AH. An asymptomatic catecholamine secreting pheochromocytoma. Am JMed.1974; 57: 953-956.

13. Manelli M, Lenders JWM, Pack K, Parent G, Eisenhofer G. Subclinical pheochromocytoma. Best Pract Res Clin Endocrinol Metab.2012; 26: 507-515.

14. Chen Y. Pheochromocytoma presenting with normal urinary catecholamine and metabolites: a case report. Formos JEndocrine Metab. 2009: 1:33-37. 